\title{
Bobillier Formula for the Elliptical Harmonic Motion
}

\author{
Furkan Semih Dündar, Soley Ersoy and Nuno T. Sá Pereira
}

\begin{abstract}
In this study, we have considered the elliptical harmonic motion which is the superposition of two simple harmonic motions in perpendicular directions with the same angular frequency and phase difference of $\frac{\pi}{2}$. It is commonly recognized that a convenient formulation for problems in planar kinematics is obtained by using number systems. Here the elliptical numbers are used to derive the Bobillier formula with two different methods for aforesaid motion; the first method depends on the Euler-Savary formula and the second one uses the usual relations of the velocities and accelerations.
\end{abstract}

\section{Introduction}

In the planar motion of two conjugate curves on one another, Euler-Savary formula gives the radius of curvature and center of path traced by a point, $[1,2]$. The complex number approach is an efficient technique which takes care of signs automatically rather than combined graphical and analytical methods. The complex number forms of Euler-Savary formula has been derived by [9]. Moreover, by using Müller's method in complex plane Euler-Savary formula has been given in [7]. From this aspect the generalization of Euler-Savary formula in Euclidean, Lorentzian, and Galilean planes has been occurred in [5]. Also, in [8] Euler-Savary formula in the case of elliptical harmonic motion has obtained using the formalism of elliptic numbers.

Key Words: Elliptical numbers, elliptical harmonic motion, Bobillier formula.

2010 Mathematics Subject Classification: 53A17, 53B50.

Received: February, 2017.

Revised: .

Accepted: . 
Here we aim to derive the Bobillier formula using the same formalism. Bobillier formula gives the relation of curvatures of second order planar motion which has been studied by [4] in two different ways and the complex number technique has been applied to obtain this formula in [3]. Bobillier formula is an analytical approach as Bobillier construction produces a geometric approach. To introduce Bobillier's construction in Euclidean plane consider the motion of the moving centrode, as it rolls over the fixed centrode by utilizing a convex concave contact and convex convex contact, momentarily rotating about the instant center $I$. The path tangent and path normal are designed by $t$ and $n$, respectively. Now consider an inclined ray $\left(\theta\right.$-ray) through $I . J_{1}\left(J_{2}\right)$, which is an inflection point, is a point of the plane which at the moment is going through a point of inflection of its path with respect to the fixed plane. The path of every inflection point, as a point of the mobile plane, has a second order contact with its path tangent. $X^{\prime}{ }_{1}\left(X^{\prime}{ }_{2}\right)$ is the center of path curvature of an arbitrary point $X_{1}\left(X_{2}\right)$ of the plane. The Bobillier construction is useful for find the fourth point on a $\theta$-ray, when any three of the following four points are known: the instant center $I$, an arbitrary point $X_{1}\left(X_{2}\right)$ of mobile plane, the inflection point $J_{1}\left(J_{2}\right)$ and the center of curvature $X^{\prime}{ }_{1}\left(X^{\prime}{ }_{2}\right)$ of the path described by $X_{1}\left(X_{2}\right)$ in the fixed plane as the moving centrode rolls on the fixed centrode; all are on the $\theta$-ray. Bobillier's construction for finding the inflection point $J_{1}\left(J_{2}\right)$ when $I, X_{1}\left(X_{2}\right)$ and $X^{\prime}{ }_{1}\left(X^{\prime}{ }_{2}\right)$ are known on the ray $I X_{1}\left(I X_{2}\right)$ is clearly shown on Figure 1 and the readers are referred to $[9,10]$.

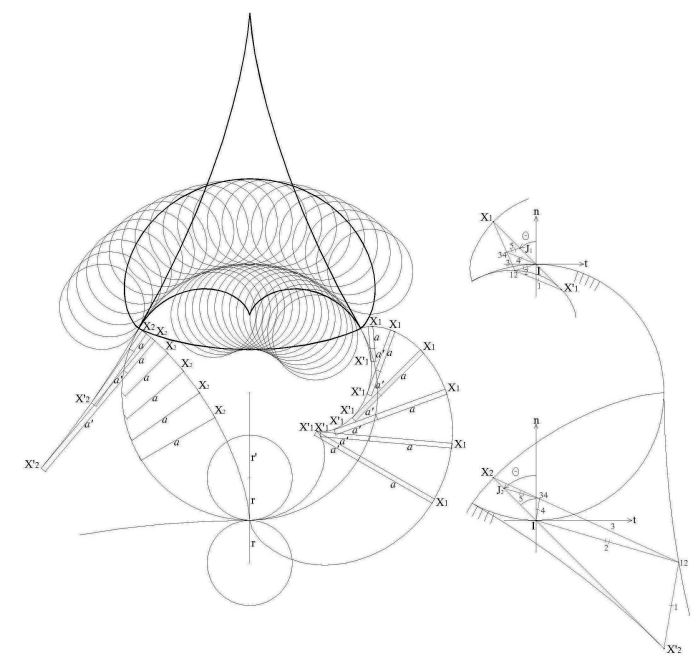

Figure 1. Bobillier's construction in Euclidean plane $E^{2}$. In the special case of an ellipse with zero eccentricity and frequency null. 


\section{Preliminaries}

This is a study expanding aspects of [8] which deals with superposition of two simple harmonic motions in perpendicular directions with the same angular frequency and phase difference of $\pi / 2$. If a point has two component simple harmonic motions in any directions with the same periods the resultant motion of the point will be harmonic in an ellipse. Such motion is called elliptical harmonic motion. A mass attached to end of a spring in two dimensions also exhibit this feature depending on the initial velocity. On the contrary, although planets around the Sun revolve in ellipses their motion is not harmonic.

In [8] authors represented elliptical harmonic motions in terms of elliptic numbers and elliptical trigonometry.

Any elliptic number is given in the form of $x+i y$ such that $x, y \in \mathbb{R}$ and it is the special case of the generalized complex number when $i^{2}=p<0$. If $p=-1$ then the elliptical number is reduced to ordinary complex number, [11]. The norm of an elliptic number $x+i y$ is given by $\|x+i y\|_{p}=\left(x^{2}-p y^{2}\right)^{1 / 2}$. The exponential of a purely elliptic number is given similar to the complex case as

$$
e^{i \theta}=\operatorname{cosp} \theta+i \operatorname{sinp} \theta
$$

however here elliptical versions of trigonometric functions are defined $\operatorname{cosp} \theta=$ $\cos (\theta \sqrt{|p|})$ and $\sin p \theta=\sin (\theta \sqrt{|p|}) / \sqrt{|p|},[6]$.

The cross product of two elliptic numbers $u=\rho_{u} e^{i \theta_{p}}$ and $v=\rho_{v} e^{i \theta_{v}}$ can defined as

$$
u \times v=\operatorname{Im}(\bar{u} v)=\rho_{u} \rho_{v} \operatorname{sinp}\left(\theta_{v}-\theta_{u}\right)
$$

or in form of

$$
u \times v=\|u\|_{p}\|v\|_{p} \operatorname{sinp}\left(\theta_{v}-\theta_{u}\right)
$$

where an overbar denotes the usual complex conjugation. This definition will be useful when we derive the Bobillier formula in the next section.

Also, the inner product of two elliptic numbers was given in [6] as follows:

$$
\langle u, v\rangle=\operatorname{Re}(\bar{u} v)=\|u\|_{p}\|v\|_{p} \operatorname{cosp}\left(\theta_{v}-\theta_{u}\right) .
$$

In [8] by considering these basic notions and handling the mobile elliptical planes $A, E$ and fixed elliptical planes $E^{\prime}$ with coordinate systems $\left\{B ; a_{1}, a_{2}\right\}$, $\left\{O ; e_{1}, e_{2}\right\}$ and $\left\{O^{\prime} ; e^{\prime}{ }_{1}, e^{\prime}{ }_{2}\right\}$, respectively, the Euler-Savary formula for elliptical harmonic motions was given as follows.

Theorem 2.1. Let $E$ and $E$ be mobile and fixed elliptic planes. A point $X$ on $E$ in a elliptical movement draws a trajectory in plane $E^{\prime}$ for which the curvature centre is at point $X^{\prime}$. In the reverse movement $X^{\prime}$ on $E^{\prime}$ draws a 
trajectory in plane $E$ for which the curvature centre is at point $X$. The relation between points $X$ and $X$ is

$$
\left(\frac{1}{a^{\prime}}-\frac{1}{a}\right) e^{-i(\alpha+2 \pi f t)}=-i\left(\frac{1}{r^{\prime}}-\frac{1}{r}\right)
$$

where $f$ is the frequency of elliptical harmonic motion, $r$ and $r^{\prime}$ are the curvatures of the pole curves. Also, a and $a^{\prime}$ represent the distances from the rotation pole to the point $X$ and $X^{\prime}$, respectively, [8].

For that purpose $\phi$ and $\psi$ were considered such as the elliptical angles that correspond to initial phase differences of the motions $A / E$ and $A / E^{\prime}$. This formula is satisfied when the abscissae of the fixed point $X$ and the moving point $X^{\prime}$ are coincident, that is, $x_{1}=x_{1}^{\prime}$.

In this study we need the general form of the Euler-Savary formula of elliptical harmonic motion which can be derived by direct calculations as

$$
\left(\frac{1}{a^{\prime}}-\frac{1}{a}\right) \operatorname{sinp}(\alpha+2 \pi f t)=\frac{1}{r^{\prime}}-\frac{1}{r} .
$$

The argument of the function sinp should be understood as an elliptical angle. Particularly if $i^{2}=-1$ and $t=0$, the equation (5) completely reduces to the usual complex form of the Euler-Savary formula (see Figure 2).

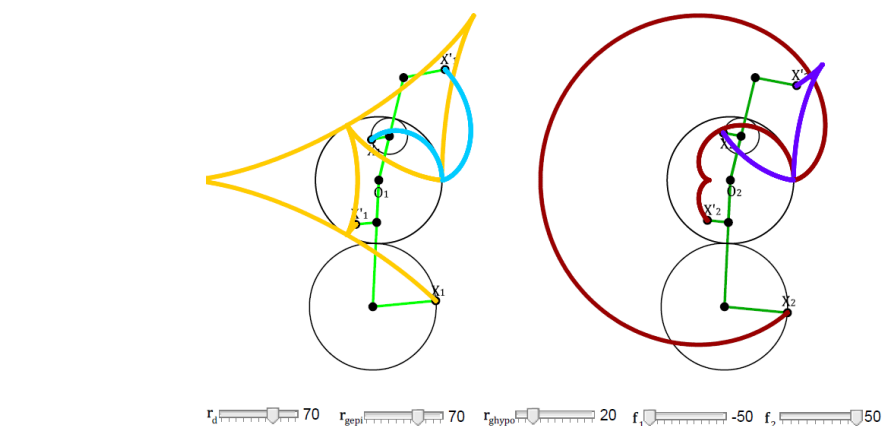

Figure 2. Euler-Savary in Euclidean plane $E^{2}$. In the special case of an ellipse with zero eccentricity.

\section{Derivation of the Bobillier formula}

In this section, we use a method to derive Bobillier formula having regard to the Euler-Savary formula (5) of elliptical harmonic motion. Let $X_{j}$ and $X_{j}^{\prime}$, $j=1,2,3$, be points linked to the mobile elliptical plane $E$ and fixed elliptical 
plane $E^{\prime}$, respectively. Suppose that $a_{j}$ and $a_{j}^{\prime}$ represent the distances from the rotation pole $I$ to the points $X_{j}$ and $X_{j}^{\prime}$, respectively. If the notation $1 / \rho_{j}=1 / a_{j}^{\prime}-1 / a_{j}$ is considered and the points $C_{j}$ are given by

$$
\overrightarrow{I C}_{j}=C_{j}=\frac{1}{\rho_{j}} e^{i \alpha_{j}} e^{i 2 \pi f t}
$$

then it is seen by virtue of Euler-Savary formula of elliptical harmonic motion with frequency $f$ and initial phase $\alpha_{j}$ that the points $C_{j}$ follows a linear orbit. Since the vectors $\overrightarrow{C_{1} C_{2}}=C_{2}-C_{1}$ and $\overrightarrow{C_{2} C_{3}}=C_{3}-C_{2}$ should be linearly dependent the cross product of the elliptic numbers vanishes as follows;

$$
C_{2} \times C_{3}+C_{3} \times C_{1}+C_{1} \times C_{2}=0 .
$$

Defining $\alpha_{j k}=\alpha_{j}-\alpha_{k}$ and substituting (3) and (6) into the last equation give us

$$
\frac{\operatorname{sinp}\left(\alpha_{32}+2 \pi f t_{32}\right)}{\rho_{2} \rho_{3}}+\frac{\operatorname{sinp}\left(\alpha_{13}+2 \pi f t_{13}\right)}{\rho_{1} \rho_{3}}+\frac{\operatorname{sinp}\left(\alpha_{21}+2 \pi f t_{21}\right)}{\rho_{1} \rho_{2}}=0 .
$$

If we product this equality by $\rho_{1} \rho_{2} \rho_{3}$, we obtain the Bobillier formula for the elliptic harmonic motion:

$$
\rho_{1} \operatorname{sinp}\left(\alpha_{32}+2 \pi f t_{32}\right)+\rho_{2} \operatorname{sinp}\left(\alpha_{13}+2 \pi f t_{13}\right)+\rho_{3} \operatorname{sinp}\left(\alpha_{21}+2 \pi f t_{21}\right)=0 .
$$

This formula exactly depends on Euler-Savary formula of elliptical harmonic motion as well as the following method reproduces the same relation directly.

\section{Alternative Derivation of the Bobillier Formula}

In this section, we give an alternative derivation of the Bobillier formula following the footsteps of [4].

$e^{i \alpha_{i}} e^{i 2 \pi f t_{j}}$ for $j=1,2,3$ are unit vectors from the rotation pole $I$ towards the points $X_{j}$ on the mobile planes for the second order elliptical harmonic motion. Since every three vectors on a plane are linearly dependent there is the following linear dependence between these vectors;

$$
\lambda_{1} e^{i \alpha_{1}+2 \pi f t_{1}}+\lambda_{2} e^{i \alpha_{2}+2 \pi f t_{2}}+\lambda_{3} e^{i \alpha_{3}+2 \pi f t_{3}}=0 .
$$


The consecutive cross products of the last equation with $e^{i \alpha_{3}+2 \pi f t_{3}}, e^{i \alpha_{1}+2 \pi f t_{1}}$ and $e^{i \alpha_{2}+2 \pi f t_{2}}$ give the coefficients as follows;

$$
\begin{aligned}
& \lambda_{1}=e^{i \alpha_{2}+2 \pi f t_{2}} \times e^{i \alpha_{3}+2 \pi f t_{3}}=\operatorname{Im}\left(\overline{e^{i \alpha_{2}+2 \pi f t_{2}}}, e^{i \alpha_{3}+2 \pi f t_{3}}\right)=\operatorname{sinp}\left(\alpha_{32}+2 \pi f t_{32}\right), \\
& \lambda_{2}=e^{i \alpha_{3}+2 \pi f t_{3}} \times e^{i \alpha_{1}+2 \pi f t_{1}}=\operatorname{Im}\left(\overline{e^{i \alpha_{3}+2 \pi f t_{3}}}, e^{i \alpha_{1}+2 \pi f t_{1}}\right)=\operatorname{sinp}\left(\alpha_{13}+2 \pi f t_{13}\right), \\
& \lambda_{3}=e^{i \alpha_{1}+2 \pi f t_{1}} \times e^{i \alpha_{2}+2 \pi f t_{2}}=\operatorname{Im}\left(\overline{e^{i \alpha_{1}+2 \pi f t_{1}}}, e^{i \alpha_{2}+2 \pi f t_{2}}\right)=\operatorname{sinp}\left(\alpha_{21}+2 \pi f t_{21}\right) .
\end{aligned}
$$

Then the linear combination of these vectors can be written with respect to the phase differences, frequency of the elliptical harmonic motion

$$
\begin{aligned}
\operatorname{sinp}\left(\alpha_{32}+2 \pi f t_{32}\right) e^{i \alpha_{1}+2 \pi f t_{1}}+ & \operatorname{sinp}\left(\alpha_{13}+2 \pi f t_{13}\right) e^{i \alpha_{2}+2 \pi f t_{2}} \\
& +\operatorname{sinp}\left(\alpha_{21}+2 \pi f t_{21}\right) e^{i \alpha_{3}+2 \pi f t_{3}}=0 .
\end{aligned}
$$

Let $V\left(X_{1}\right)$ and $J\left(X_{1}\right)$ be the velocity and acceleration vectors of point $X_{1}$ in the fixed elliptical plane $E^{\prime}$, respectively. Then the following equation holds;

$$
\frac{1}{a^{\prime}{ }_{1}-a_{1}}=\frac{\left\langle J\left(X_{1}\right), e^{i \alpha_{1}} e^{i 2 \pi f t_{1}}\right\rangle}{\left\langle V\left(X_{1}\right), V\left(X_{1}\right)\right\rangle} .
$$

Further, $J\left(X_{1}\right)$ can be decomposed to the trajectorywise invariant component, tangential acceleration component and centripetal component as follows;

$$
J\left(X_{1}\right)=J(I)+i a_{1} \dot{\omega} e^{i \alpha_{1}} e^{i 2 \pi f t_{1}}-a_{1} \omega^{2} e^{i \alpha_{i}} e^{i 2 \pi f t_{1}},
$$

where $\omega$ is the angular velocity of the elliptical harmonic motion $E / E^{\prime}$ and $J(I)$ is "acceleration of the point on $E^{\prime}$ that coincides instantaneously with $I$." Using the expansion (12) in (11) and using $V\left(X_{1}\right)=\omega a_{1}$ we obtain:

$$
\frac{\left\langle J(I), e^{i \alpha_{1}} e^{i 2 \pi f t_{1}}\right\rangle}{\omega^{2}}=\rho_{1} .
$$

In a similar manner resembling equations can be found for $X_{2}, X_{3}$ and the general result for $j=1,2,3$ is given by

$$
\frac{\left\langle J(I), e^{i \alpha_{j}} e^{i 2 \pi f t_{j}}\right\rangle}{\omega^{2}}=\rho_{j} .
$$

Finally, by the inner product of the equation (10) with $J(I) / \omega^{2}$ the following theorem is given. 
Theorem 4.1. In the elliptical harmonic motion the relations between the centers of curvatures is given by the equation

$$
\rho_{1} \operatorname{sinp}\left(\alpha_{32}+2 \pi f t_{32}\right)+\rho_{2} \operatorname{sinp}\left(\alpha_{13}+2 \pi f t_{13}\right)+\rho_{3} \operatorname{sinp}\left(\alpha_{21}+2 \pi f t_{21}\right)=0
$$

which is called Bobillier Formula for the elliptical harmonic motion.

\section{Conclusion}

In this study, we derived the Bobillier formula for elliptical harmonic motion in terms of elliptic numbers and elliptical trigonometry. This result is a contribution to the analysis of oscillatory aspects in treating the phenomenon of motion. As a by-product of our study, we defined cross product of two elliptic numbers. We conclude that the form of outer product is a new contribution to the literature.

\section{References}

[1] Blaschke, W. and Müller, H. R., Ebene Kinematik, Verlag von R. Oldenbourg, München, 1956.

[2] Buckley, R. and Whitfield E. V., The Euler-Savary Formula, The Mathematical Gazette, 33(306), 297-299, 1949.

[3] Ersoy, S. and Bayrak, N., Bobillier Formula for One Parameter Motions in the Complex Plane, J. Mechanisms Robotics, 4(2), 24501[4 pages], 2012.

[4] Fayet M., Bobillier Formula as a Fundamental Law in Planar Motion, ZAMM Z. Angew. Math. Mech., 82(3), 207-210, 2002.

[5] Gürses, N., Akbiyik, M. and Yüce, S., One-Parameter Homothetic Motions and Euler-Savary Formula in Generalized Complex Number Plane $C_{J}$, Adv. Appl. Clifford Algebras 26 (2016), 115-136.

[6] Harkin, A. A. and Harkin, J. B., Geometry of Generalized Complex Numbers, Math. Mag., 77, 118-129, 2004.

[7] Masal, M., Tosun, M. and Pirdal, A. Z., Euler Savary Formula for the One Parameter Motions in the Complex Plane $\mathbb{C}$, Int. J. of Physical Sci., 5(1), 6-10, 2010.

[8] Pereira, N. T. Sá. and Ersoy, S., Elliptical Harmonic Motion and EulerSavary Formula, Adv. Appl. Clifford Algebras 26 (2016), 731-755. 
[9] Sandor, G. N., Erdman, A. G., Hunt, L. and Raghavacharyulu, E., New Complex-Number Forms of the Euler-Savary Equation in a Computeroriented Treatment of Planar Path curvature Theory for Higher-Pair Rolling Contact, ASME J. Mech Des., 104, 227-238, 1982.

[10] Sandor, G. N. and Erdman, A. G., Advanced Mechanism Design: Analysis and Synthesis, Vol.2. Prentice-Hall, Englewood Cliffs, NJ., 1984.

[11] Yaglom, I. M., Complex Numbers in Geometry, Academic Press, New York, 1968.

Furkan Semih DUNDAR,

Department of Physics,

Bogazici University,

34342 Bebek, Istanbul, Turkey.

Email: f.semih.dundar@yandex.com

Soley ERSOY,

Department of Mathematics,

Sakarya University,

Serdivan, Sakarya, Turkey

Email: sersoy@sakarya.edu.tr

Nuno T. Sá Pereira

Nuno T. Sá PEREIRA,

Portugal.

Email: sa.pereira.nuno@gmail.com 